\title{
The Study of Distribution Data Characteristics of Cultural Creativity Industry Agglomeration Areas Supported by Historic Districts in Jiangsu Province
}

\author{
Yujie Xin and Rong Han
}

\begin{abstract}
Based on the research on the distribution of cultural creativity industry agglomeration areas supported by historic districts in Jiangsu Province, this paper studies the distribution data of this typed agglomeration areas from the perspective of gathering region, type, and function mode, using the quantitative and non-quantitative method, as well as the combination of subjective and objective methods. Also, it arrives the conclusion of "three cycles with one area" combined with the distribution affecting factors of this typed areas, and provides urban planning information system with the basic data support.
\end{abstract}

Index Terms-Cultural creativity industry agglomeration areas, distribution data characteristics, distribution trends, historic districts.

\section{INTRODUCTION}

Today, the cultural creativity industry has become a major highlight in the development of all cities, but there still lacks the data analysis about its distribution in urban planning information system, which will affect the scientific and rational urban design.

Currently, foreign studies on questions about the cultural creativity industry mainly focus on its definition, the internal corporate behavior, the creativity class, the creativity industry agglomeration area and so on. Meanwhile the western developed countries have established a number of famous historic-district typed cultural creativity industry agglomeration areas. Compared to foreign studies, related domestic studies are still in its initial stage. Domestic studies mainly focus on the macro-level discussions on creativity industry agglomeration areas, and at the micro level, related studies are really rare, especially for the agglomeration area in the form of a certain carrier.

The selection of the cultural creativity industry agglomeration area in the form of historic districts as the study object is of great significance of keeping pace with the times. Jiangsu Province is faced with the industrial restructuring brought by accelerating the transformation of economic growth, and it has clearly defined the focus on the development of culture industry in its Twelfth Five-year Plan. Meanwhile, the rich cultural content in Jiangsu Province provides favorable conditions for the

Manuscript received February 5, 2015; revised June 1, 2015. This work was financially supported by culture research project "Research on Image Integration and Environment Renovation Methods of Historical Villages in Zhenjiang City" of Jiangsu province (Project No.:13YB24).

The authors are with the Jiangsu University, Zhenjiang city, Jiangsu Province, China (e-mail: 1223358505@qq.com, 33315935@qq.com). agglomeration of cultural creativity industry. Therefore, the choice of cultural creativity industry agglomeration areas supported by historic districts in Jiangsu Province as the research object is able to provide the complete basic urban geographic information data for the planning.

\section{The AgGlomeration OF Historic DistrictS AND CULTURAL CREATIVITY INDUSTRY}

\section{A. Historic Districts}

The term "historic district" was initially from the 1933 international conference in Athens, and then a series of international conferences and international regulations clearly put forward the significance of protecting historic districts [1]. In 1986, China's State Council formally proposed the concept of the historic district, namely the district or buildings which own relatively concentrated cultural relics and historic sites, or can fully reflect the traditional style and national or local features of a particular historical period [2]. It is an important area to reflect the urban features, personality and temperament, which both involves the "objective forms", and the "intangible culture" reflecting the special values of historic districts. Meanwhile, the social attributes of historic districts include the resident groups and the formed social structure form, which constitutes the "subject form" of historic districts together with "intangible culture"[3].

The cultural creativity industry is established based on cultural factors. And the creativity industry agglomeration in different regions will present their uniqueness due to differences in the regional culture, and this uniqueness will strengthen their competitiveness [4]. Historic districts will provide material, human and social resources for the cultural creativity industry agglomeration, and lay the foundation for the spatial organization model.

\section{B. The Cultural Creativity Industry Agglomeration}

In China, the cultural creativity industry agglomeration area is defined as: "The area that gathers a certain number of cultural creativity enterprises, and is equipped with a certain scale of industry, independent creativity capability, and specialized services and public service platform; and can provide appropriate infrastructure and public services" [5]. Cultural creativity industry has a strong agglomeration tendency in space, and the characteristics of its working process determine the industry of this kind to heavily depend on diversified information, and it usually forms a unique spatial environment in some places in the urban. It is like a cultural symbol of the city, which not only creates 
huge profits, but also contributes to the continuation of the urban context. The urban context is the unique charm and value of the city, and if the context fractures, the city's original cultural accumulation and inheritance will disappear, resulting in the loss of the city's profound cultural connotation and spirit [6]. And the cultural creativity industry agglomeration areas developed on the basis of historic districts can better reshape the space image of the historic district, and shape the city's characteristic landscape, as well as enhance the urban brand.

\section{Distribution DATA CHARACTERISTICS OF CULTURAL CREATIVITY INDUSTRY AGGLOMERATION AREAS SUPPORTED BY HistORIC DisTRICTS IN JIANGSU PROVINCE}

Through the all-around investigation on cultural creativity industry agglomeration areas supported by historic districts in Jiangsu Province, we can discuss the distribution data characteristics of this kind of area from the following perspectives, combined with data organization and analysis.

\section{A. Geographical Distribution Characteristics}

Based on the actual situation of Jiangsu Province, the agglomeration area of this kind can be divided into Northern Jiangsu, Central Jiangsu and Southern Jiangsu in the large geographical range. In addition, the distribution characteristics can also be analyzed in the scale level of city.

\section{1) Distribution characteristics of Northern Jiangsu, Central Jiangsu, and Southern Jiangsu}

a) Quantitative analysis

Table I is made based on the material collection of this typed agglomeration areas in Jiangsu Province. We can see from the Figure that, there are 25 agglomeration areas in Northern Jiangsu, 14 in Central Jiangsu and 12 in Southern Jiangsu.

TABLE I: REGION DISTRIBUTION STATUS OF CULTURAL CREATIVITY INDUSTRY AGGLOMERATION AREAS SUPPORTED BY HISTORIC DISTRICTS IN JIANGSU PROVINCE

\begin{tabular}{|c|c|c|c|}
\hline $\begin{array}{l}\text { Distribution } \\
\text { region }\end{array}$ & Quantity & City & Name \\
\hline $\begin{array}{l}\text { Southern } \\
\text { Jiangsu }\end{array}$ & 25 & Suzhou & $\begin{array}{l}\text { Pingiiang Road, Shantang } \\
\text { Street, Luzhi, Changmen, } \\
\text { Zhouzhuang, Tongli, } \\
\text { Dongshan, Luxiang, } \\
\text { Sulun Textile Mill, } \\
\text { Suzhou Match Factory, } \\
\text { Taihe Flour Mill, Suzhou } \\
\text { First Silk Factory, Suzhou } \\
\text { Valve Factory, Suzhou } \\
\text { Sandalwood Fan Factory, } \\
\text { Wuxian Embroidery } \\
\text { Factory } \\
\text { Qing Ming Bridge, } \\
\text { Huishan Age-old Street, } \\
\text { Xiaolouxiang, } \\
\text { Rongxiang, Nanchang } \\
\text { Street, The Museum of } \\
\text { Chinese National } \\
\text { Industry and Commerce } \\
\text { (originally is Baoxing } \\
\text { Flour Mill), Huallywood } \\
\text { (originally is Xuelang } \\
\text { Rough Rolling Mill ), } \\
\text { Beicangmen Art of }\end{array}$ \\
\hline
\end{tabular}

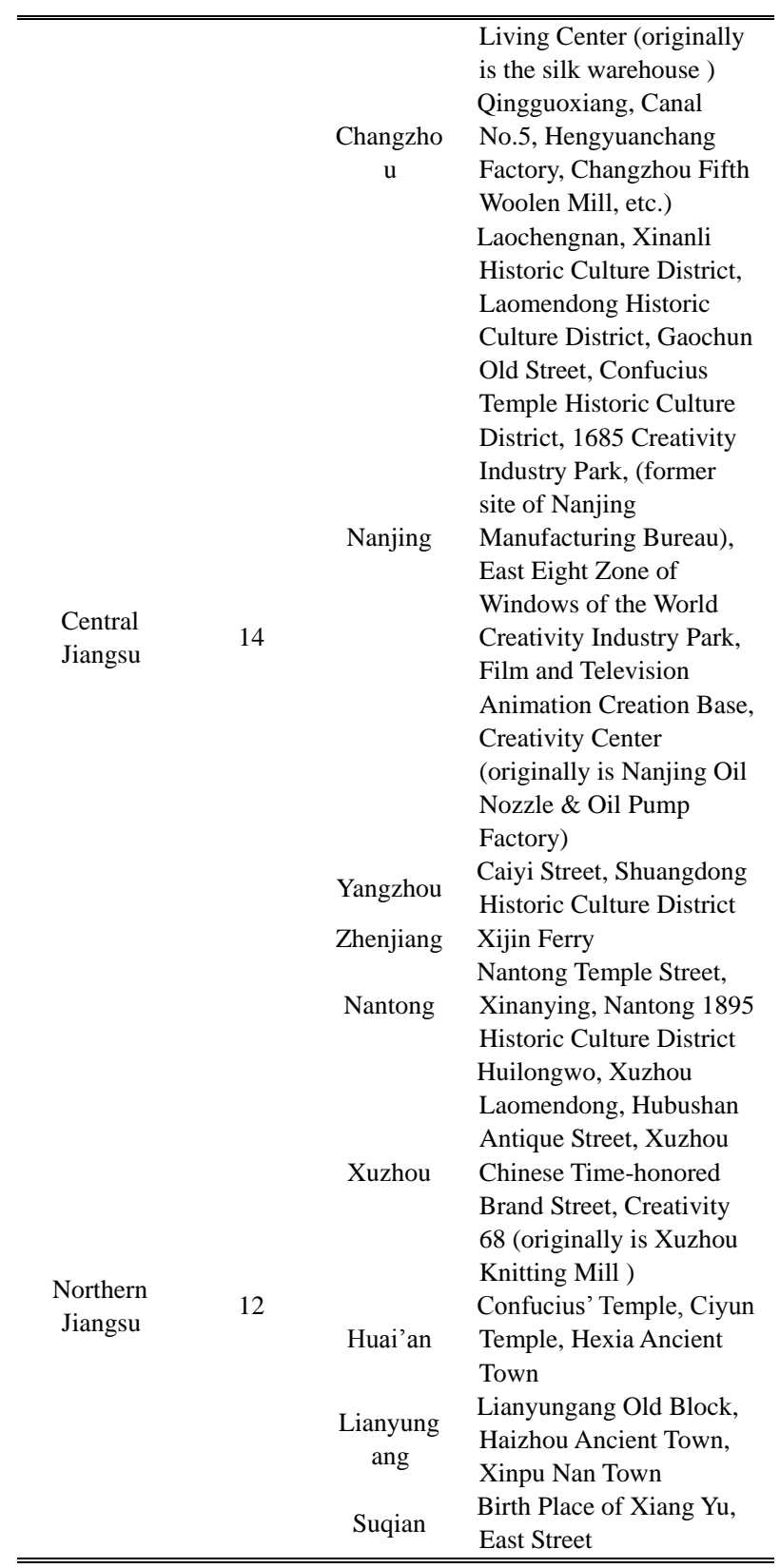

Meanwhile, combined with Fig. 1 we can visually get the distribution proportion of this typed agglomeration areas in three regions.

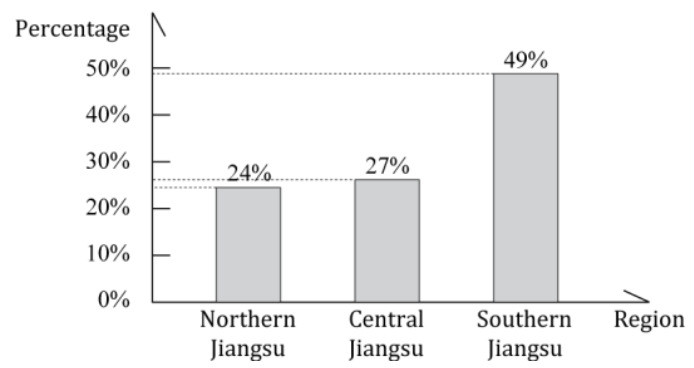

Fig. 1. The distribution proportion of cultural creativity industry agglomeration areas supported by historic districts in Northern Jiangsu, Central Jiangsu, and Southern Jiangsu.

From the data analysis above, we get that the proportion of this typed areas is of $49 \%$ in Southern Jiangsu, while the proportion in Northern Jiangsu and Central Jiangsu are $24 \%$ and $27 \%$. Therefore, Southern Jiangsu has advantages in 
distribution quantity, while the distribution quantity in Northern Jiangsu and Central Jiangsu are relatively small.

\section{b) Location analysis}

The quantity considerations in the location region of this typed agglomeration areas are not enough for the problem analysis, so the agglomeration area locations in each region are collected and analyzed.

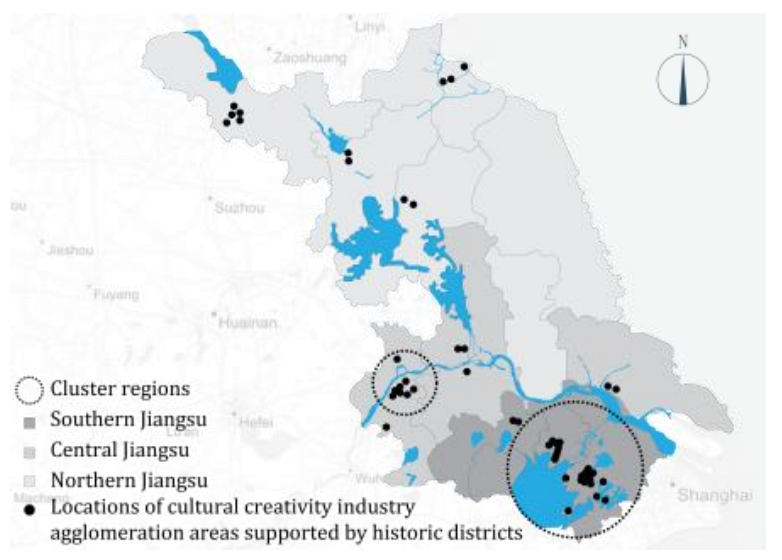

Fig. 2. Locations of cultural creativity industry agglomeration areas supported by historic districts in Northern Jiangsu, Central Jiangsu and Southern Jiangsu.

Fig. 2 shows that the distribution of agglomeration areas in Southern Jiangsu is centralized. Although there are some scattered locations, the overall shape is clustered with a high intensity. For Central Jiangsu, the distribution of agglomeration areas is relatively fragmented, but also presents the emergence of groups with a non-weak intensity. And in Northern Jiangsu, the distribution of agglomeration areas is scattered with a round shape, so the correlation between different agglomeration areas is relatively poor.

2) Distribution characteristics within the city

\section{a) Quantitative analysis}

It is necessary to make a detailed analysis of this typed agglomeration areas with the narrowed region scope based on the analysis on a wide range scope. Therefore, on the basis of Table 1, we get the following results through the distribution quantity statistics of this typed agglomeration areas in their located cities: there are 15 agglomeration areas in Suzhou, 8 in Wuxi, 2 in Changzhou, 9 in Nanjing, 2 in Yangzhou, 1 in Zhenjiang, 2 in Nantong, 5 in Xuzhou, 2 in Huai' an, 3 in Lianyungang, and 2 in Suqian. And Fig. 3 is made based on the further data analysis.

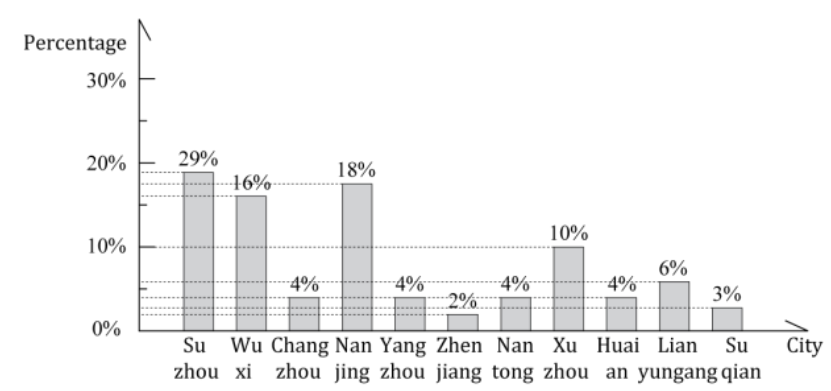

Fig. 3. The distribution quantity of cultural creativity industry agglomeration areas supported by historic districts in different cities of Jiangsu Province.

As is shown in Fig. 3, the distribution quantity of this typed agglomeration areas in Suzhou is $29 \%$, ranking first in the Province; followed by Nanjing, the distribution quantity of which is $18 \%$; then is Wuxi with the distribution quantity of $16 \%$. And the distribution quantity of other cities is $\leq 10 \%$, especially Suqian and Zhenjiang, the distribution quantity of which is $3 \%$ and $2 \%$.

\section{b) Location analysis}

Fig. 4 shows the distribution characteristics of this typed agglomeration areas in various cities of Jiangsu Province.

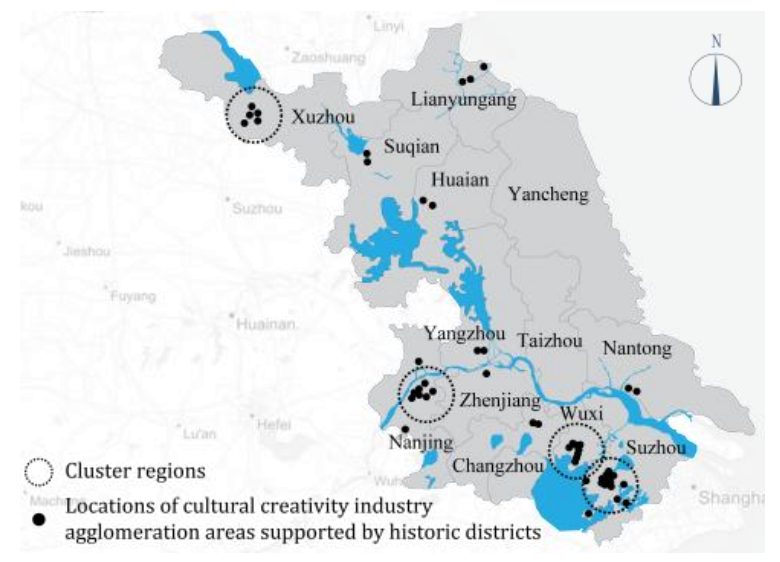

Fig. 4. The distribution locations of cultural creativity industry agglomeration areas supported by historic districts in various cities of Jiangsu Province.

Fig. 4 shows that the distribution of this typed agglomeration areas in Suzhou, Wuxi, Nanjing and Xuzhou is clustered, and most of the clustered regions are near rivers. Among them, the clustered density in Suzhou is the highest, followed by Wuxi, Nanjing and Xuzhou. On the whole, the clustered regions are unevenly distributed, and for other cities, they are of fewer agglomeration areas, failing to form a group. At the same time, there is relevance between agglomeration areas in Suzhou and Wuxi, but the relevance among other cities is relatively weak.

\section{B. Typological Distribution Characteristics}

The type of cultural creativity industry agglomeration areas can be divided into two categories: one uses the traditional districts as the space carrier for the development of creativity industry (hereinafter it will be simply referred as the traditional district); another is transformed from historic industrial area (hereinafter it will be simply referred as historic industrial area). Now we will analyze these two types of cultural creativity industry agglomeration areas in the aspect of distribution quantity and locations.

\section{1) Quantitative analysis}

Based on Table I, we get the following data through the statistics of the distribution quantity of these two types of cultural creativity industry agglomeration areas: there are 34 traditional districts and 17 historic industrial areas, which will be shown in Fig. 5 with more visualized distribution features.

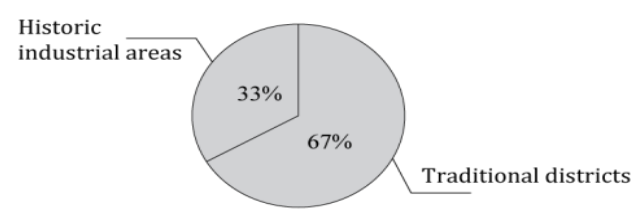

Fig. 5. The distribution quantity of these two types of agglomeration areas in Jiangsu Province. 
As shown in the above Figure, the distribution quantity of traditional districts in Jiangsu Province is $67 \%$, while the distribution quantity of historic industrial areas is only $33 \%$. Therefore, in Jiangsu Province, the distribution quantity of traditional districts is more than that of historic industrial areas.

\section{2) Location analysis}

On the basis of the distribution quantity analysis of these two types of agglomeration areas, we still need to make statistics of their distribution locations to obtain their distribution characteristics.

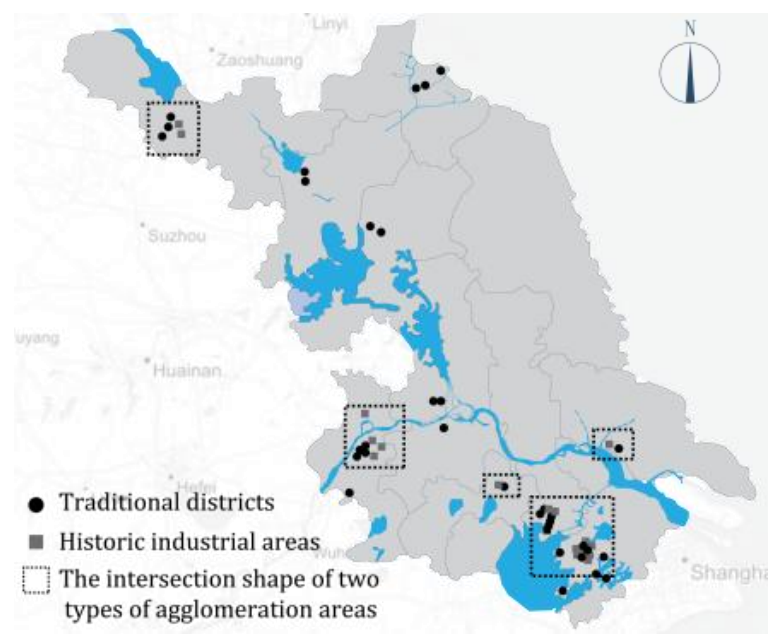

Fig. 6. The intersection shape of two types of agglomeration areas.

As is shown in Fig. 6, there are intersections of two types of agglomeration areas. Usually the appearance of historic industrial areas is accompanied by traditional industrial areas. And, in the high-density cities, Suzhou and Wuxi, there are larger intersections of two types of agglomeration areas. We can see that these two types of agglomeration areas are of interdependence to some extent.

\section{Functional Distribution Characteristics}

Combined with the actual situation, this typed agglomeration areas in Jiangsu Province can be roughly divided into three modes from the perspective of features. 1) Agglomeration areas of Mode I: take tourism, culture and business as the main features, and residence as an auxiliary feature. 2) Agglomeration areas of Mode II: take the film and television production as the main feature. 3) Agglomeration areas of Mode III: take art creation, design and exhibition as the main features, and business as the auxiliary feature.

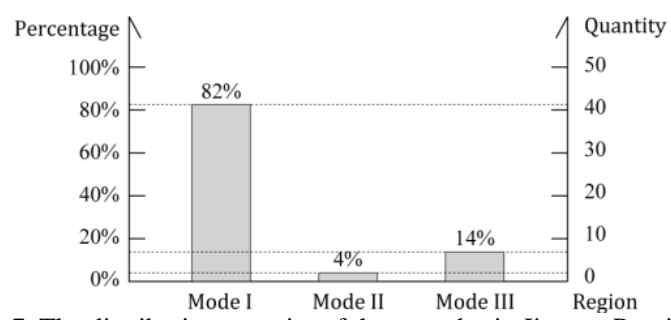

Fig. 7. The distribution quantity of three modes in Jiangsu Province.

\section{1) Quantitative analysis}

As is shown in Fig. 7 made on the basis of Table I, there are 42 agglomeration areas of Mode I in Jiangsu Province, accounting for $82 \%$ of the total; 2 of Mode II accounting for
$4 \%$ of the total; 7 of Mode III, accounting for $14 \%$ of the total. Therefore, we can conclude that in Jiangsu Province, this typed agglomeration areas are more inclined to keep the history and culture of the historic districts, but lack flexibility and richness in the features of agglomeration areas.

\section{2) Location analysis}

Based on the distribution material analysis of three modes of agglomeration areas, we obtain their region distribution situation, as is shown in Fig. 8.

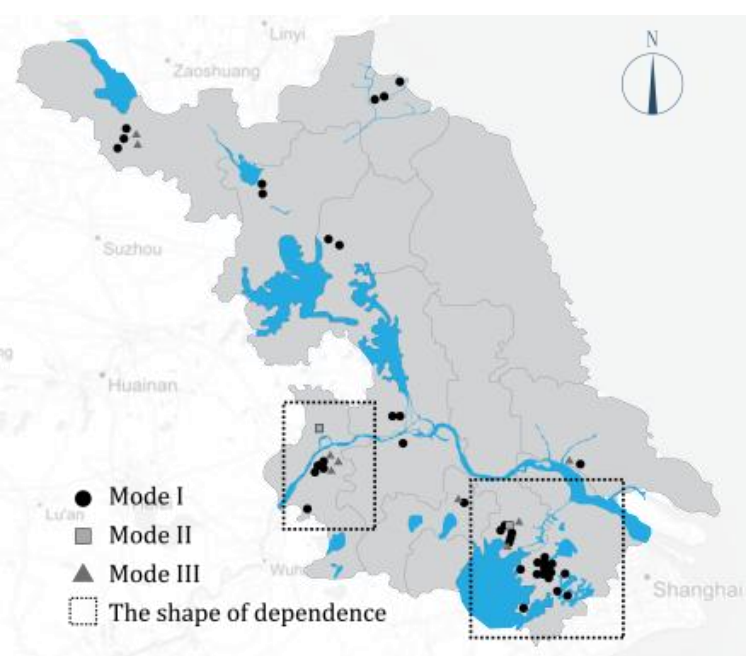

Fig. 8. The region distribution of three modes.

As is shown in the Figure, the distribution of Mode II and Mode III depends on Mode I. And in the clustered area of Mode I, there is a high appearance rate of Mode II and Mode III, which can form a larger group together with Mode I. Also, if we ignore the existence of Mode I, the distribution of Mode II and Mode III is slightly scattered with a low clustering degree, especially Mode II. Therefore, the core features of cultural creativity industry agglomeration areas supported by historic districts in Jiangsu Province are still tourism and culture based.

\section{Distribution TREND OF CUlTURAl CREATIVITY INDUSTRY AGGLOMERATION AREAS SUPPORTED BY Historic DisTRICTS IN JIANGSU PROVINCE}

To sum up, the distribution mode of this typed agglomeration areas can be roughly concluded as "three circles with one area", as shown in Fig. 9.

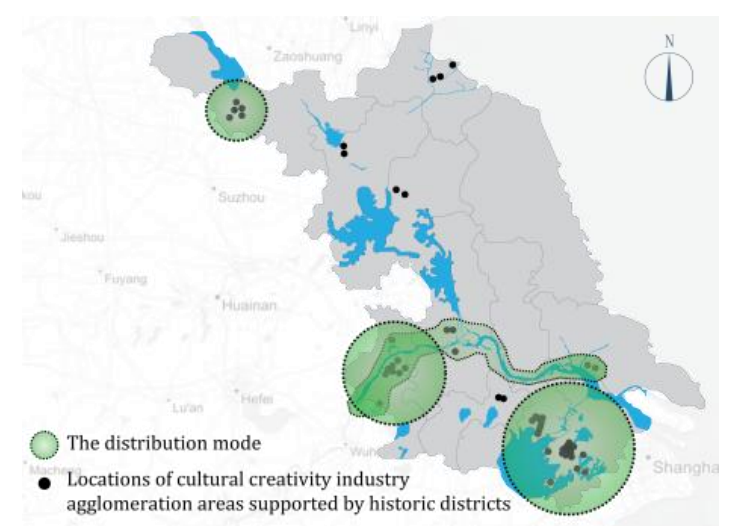

Fig. 9. The distribution mode of "three circles with one area". 


\section{A. Three Circle-Based Distribution Trend}

Three circles refer to the distribution circle around Taihu Lake, Confucius Temple-centered distribution circle and historic culture distribution circle of Old Xuzhou.

\section{1) The distribution circle around taihu lake}

Taihu Lake is the largest lake in East China, and there has formed a City Group around it. There are good talent, physical, social and cultural resources in this distribution circle, which are all conditions needed by this typed agglomeration areas. In the distribution circle around Taihu Lake, this typed agglomeration areas present the advantage of quantity, quality and intensity. At the same time, they also have a wealth of content, which covers the agglomeration area of traditional historic district and historic industrial area with three modes and help to create a more flexible urban space.

\section{2) Confucius temple-centered distribution circle}

Confucius Temple-centered distribution circle is not inferior in all respects. Its located city, Nanjing is the capital city of Jiangsu Province, which is of great advantages in economics, politics, transportation and other aspects; meanwhile Nanjing is one of China's four ancient capitals and is an important birthplace of Chinese civilization. In this distribution circle, the distribution of this typed agglomeration areas is not only of a large number, but also forms into groups; also there are three modes of agglomeration areas in this circle. The weak development of this typed agglomeration areas in this circle lies in their failure in forming a good relevance with the surrounding cities, so the distribution of this typed agglomeration areas is of a weak clustering, but with a not bad development quality.

\section{3) Historic culture distribution circle of old Xuzhou}

Compared with the first two distribution circles, the development of historic culture distribution circle of Old Xuzhou is weak. As an important gateway in East China, Xuzhou is known as one of China's Nine States with convenient transportation and other material resources, which provides the possibility for the development of this typed agglomeration areas. However, the shortcomings of this distribution circle are that this typed agglomeration areas in this distribution circle are of small number with a single type and mode, and though their quality is poor, they have presented the initial trend of clustering.

\section{B. The Distribution Trend of "One Area"}

"One Area" refers to the middle and lower reaches of Yangtze River. The development of a city always has something to do with rivers, and there are city groups with a high development in the middle and lower reaches of Yangtze River in Jiangsu Province. With the development of the city, its political, economic, transportation, historical and cultural resources show a huge advantage. All these will contribute to the occurrence and development of the cultural creativity industry agglomeration areas supported by historic districts. Therefore, in this "One Area", there is a development trend of gathering along the Yangtze River. And it shows the advantage in quantity with richness and flexibility in the type and model. But its intensity is not high, because the most of the passing areas of Yangtze River are junctions of cities, so their development is relatively weak; when Yangtze River flows through Nanjing, we can see the advantages it provides for the development of this typed agglomeration areas.

\section{CONCLUSIONS}

As an emerging industry, the cultural creativity industry agglomeration areas supported by historic districts in Jiangsu Province are still in their initial stage, so there are less data statistics and analysis on them, which will further affect the improvement of the urban planning information systems and the promotion of decision-making and management level. And the full range of information collection and conversion will better serve the urban construction and development.

\section{REFERENCES}

[1] H. L. Yang, "Discussion of the protective development of Guangzhou historic districts," Journal of Urban Problems, vol. 5, pp. 23-24, Sept. 1998.

[2] Y. Chen, "When history meets creativity - The preliminary analysis of China's current agglomeration process of cultural creativity industry supported by historic districts," Journal of Southeas Academic Research, vol. 2, pp. 111-120, Mar. 2012.

[3] S. J. Li, "Historic districts environmental reform model - Xintiandi in Shanghai, Suzhou Tong Fang Avenue as an example," Unpublished master dissertation, Nanjing Forestry University, Nanjing, 2007.

[4] Y. P. Jin, An Introduction to Cultural Creativity Industry, Beijing: Higher Education Press, 2010, pp. 196.

[5] Y. Chen, "When history meets creativity - The preliminary analysis of China's current agglomeration process of cultural creativity industry supported by historic districts," Journal of Southeast Academic Research, vol. 2, pp. 111-120, Mar. 2012

[6] W. N. Wang and P. Y. Zhang, "The creative industries and urban regeneration," Journal of Urban Planning Forum, vol. 2, pp. 22-27, Mar. 2006

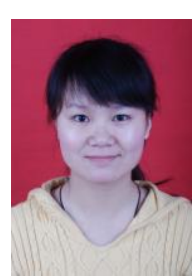

Yujie Xin was born in Henan Province, in 1989 She is currently pursuing a master degree in Jiangsu University, where is in Zhenjiang city, Jiangsu Province, China. As a master student, her research field is directed to design of urban planning information system.

She has won several contest honors associated with the major field and school scholarships since she join in the university. Not only has she been engaged in teaching assistant, but also has published couple papers one of which is the study of the curriculum structure of environmental architectural design courses. Meanwhile, she participated in Design Thinking of Cultural Creativity Industry Agglomeration Areas supported by historic districts in Jiangsu Province and other project research.

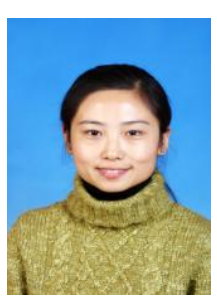

Rong Han was born in Shandong Province, in 1976. She got her Ph.D degree from Soochow University, and works as an associate professor currently in Art school of Jiangsu University. Her research direction is environmental resource integration and information processing.

She has published more than 30 professional papers, and four monographs. The publisher is respectively SDX Joint Publishing Company, The Commercial Press and People`s Daily Press. 